\title{
Clinical Efficacy and Prognostic Factors of Chemoreduction Combined with Topical Treatment for Advanced Intraocular Retinoblastoma
}

\author{
Yan Liu ${ }^{1 \&}$, Xi Zhang ${ }^{2 \&}$, Fang Liu ${ }^{1 *}$, Ke-Lei Wang ${ }^{3}$
}

\begin{abstract}
Objective: To explore the clinical efficacy and prognostic factors of chemoreduction combined with topical treatment of advanced intraocular retinoblastoma (RB). Materials and Methods: A total of 22 eyes from 17 children with RB were selected for the study and treated with chemoreduction combined with topical cryotherapy, transpupillary thermotherapy (TTT) or episcleral plaque brachytherapy. Clinical and follow-up data were retrospectively analyzed. Results: All children received $2 \sim 6$ courses of chemoreduction treatment, $(4.5 \pm 0.8$ courses on average); 17 eyes from 13 children were treated by chemoreduction combined with cryotherapy or TTT and 5 eyes from 4 children with chemoreduction combined with ${ }^{125}$ I episcleral plaque brachytherapy. The eye retention rate was $81.8 \%(18 / 22)$, among which $38.9 \%(7 / 18)$ featured restored or maintained good vision. Postoperative follow-up period was 7 to 34 months, $(18.6 \pm 5.2$ months on average). The recurrence rate was 41.2\% (7/17), among which $57.1 \%$ (4/7) were controlled by supplementing or appending cryotherapy or TTT treatment during the follow-up period. The tumor basal diameter and thickness were significantly reduced $(P<0.05$ or $P<0.01)$ after treatment. All children demonstrated different degrees of hair loss, $70.6 \%(12 / 17)$ with different degrees of gastrointestinal reactions, $5.88 \%(1 / 17)$ with neutropenia and $11.8 \%(2 / 17)$ being seriously infected during the chemotherapeutic treatment. Univariate and logistic regression analysis showed that tumor basal diameter before treatment had a significant effect on the prognosis $(P<0.01)$. Conclusions: Chemoreduction combined with topical therapy can effectively control RB in the short term, and tumor basal diameter before treatment is an independent risk factor for prognosis.
\end{abstract}

Keywords: Retinoblastoma - chemoreduction - cryotherapy - transpupillary thermotherapy - brachytherapy - plaque

Asian Pac J Cancer Prev, 15 (18), 7805-7809

\section{Introduction}

Retinoblastoma $(\mathrm{RB})$ is a kind of embryonic malignant tumor originated from the primitive stem cells in nuclear layer of the retina, the most common intraocular malignant tumor in infants, and a serious harm to children's vision and lives (Shields et al., 2010). The incidence of RB in infantile tumors reaches up to $4 \%$, both in one and two eyes, among which monocular incidence accounts for about $60 \% \sim 80 \%$. The primary goal of treating RB is to save children's lives, followed by preservation of eyeballs, and then the consideration of useful vision. Treatments vary from person to person. Trend of metastasis, risks of second malignancy, sizes and locations of the tumors, systemic conditions, visual prognosis estimates and other specific situations should be taken into consideration comprehensively. Currently, the common treatments include ophthalmectomy, evisceration, external beam radiation therapy, chemoreduction, transpupillary thermotherapy (TTT), cryotherapy, laser photocoagulation, episcleral plaque brachytherapy (Peterson et al., 2011). However, conventional therapeutic regimens such as ophthalmectomy and evisceration cause not only the highest rate of overall survival (OS) but also the deformities and poor outcomes in children after surgery, and reduce their quality of life (QOL) (Huang et al., 2013). In recent years, some developed countries gradually use chemotherapy as the first-line therapy instead of traditional therapeutic regimens to avoid the trauma and pain caused by traditional therapeutic regimens, which greatly improves the OS rate and QOL of children. In this study, children with advanced intraocular RB were treated by chemoreduction combined with topical treatment, and the clinical follow-up data was retrospectively analyzed as follows.

${ }^{1}$ Nanjing University of Chinese Medicine, Nanjing, ${ }^{3}$ Department of Ophthalmology, Wuxi Hospital of Traditional Chinese Medicine, Wuxi, Jiangsu, China, ${ }^{2}$ Russian Cancer Research Center of Russian Academy of Medical Sciences, Moscow, Russia ${ }^{\star}$ Equal contributors *For correspondence: Ler.30ler3000@163.com 


\section{Materials and Methods}

\section{General data}

A total of 22 eyes from 17 children admitted in Department of Ophthalmology in the 81 Hospital of Nanjing City and Federal state institution "Russian cancer research center them.Blokin" Russian academy of medical sciences from Feb. 2011 to Apr. 2013 were selected, among which 13 eyes from 10 boys and 9 from 7 girls; the age range was 7 84 months with average age being $(28.65 \pm 5.76)$ months; the body weight ranged from $7.9 \sim 20.3 \mathrm{~kg}$ with average weight being $(14.67 \pm 2.03) \mathrm{kg}$. Under the condition of conventional general anesthesia, binoculus examination including ophthalmotonometry, anterior segment examination under the operating microscope, indirect fundus examination under the ophthalmoscope was conducted to exclude neovascular glaucoma as well as refractive media opacity; imageological examinations including B-ultrasound of eyes and MRI or CT scan of eyes and skulls were performed to exclude pineoblastoma and extraocular metastasis; and systemic examinations were made to exclude abnormal hepatorenal function and hearing impairment. All of the affected eyes were conformed to the $\mathrm{V}$ period of Reese-Ellsworth (RE) taxonomy, namely advanced intraocular RB.

\section{Methods}

Chemoreduction: The current international common CEV protocol was used. Intravenous infusion of $18.60 \mathrm{mg} /$ $\mathrm{kg}$ carboplatin was administrated on $\mathrm{d} 1$, while intravenous infusion of $0.05 \mathrm{mg} / \mathrm{kg}$ vincristine and $5.00 \mathrm{mg} / \mathrm{kg}$ etoposide combined with nutriceutical, liver-protection and antiemetic drugs on d 2. Plenty of water based on body condition was provided to increase the urine output to protect the kidney function in children. The intervals of chemotherapies were 3 4 weeks, during which time blood routine, urine routine as well as all biochemical terms were reexamined.

Topical treatment: Intraocular tumors were frozen or treated by TTT under general anesthesia after 2 courses of chemoreduction. Cryotherapy was used for the treatment of smaller tumors on retinal equator and the surrounding portion, each local tumor was frozen and thawed for three times till the entire tumor was covered by ice ball under indirect ophthalmoscope. TTT therapy was used for the treatment of tumors near the posterior location of the retina, and conducted with the tumor being heated to gray or white, with power being $240 \sim 800 \mathrm{~mW}$ and spot diameter $1.2 \mathrm{~mm}$, and transmitted via indirect ophthalmoscope.

${ }^{125}$ I episcleral plaque brachytherapy: Plaque brachytherapy was used when cryotherapy and TTT treatment failed. During the month after $4 \sim 6$ courses of chemoreduction, individualized applicators were customized according to the size and location of the tumors as well as conditions of cornea, vitreum and retina. Affected eye conjunctiva was incised under general anesthesia to examine the thickness of the sclera and tissues outside the sclera, while transillumination was used to localize the tumor and make acrylic acid transparent to simulate applicators on the sclera surface, and then the tumor shadow was labeled. Lamellar sclera nylon suture was preseted, simulated sclera applicator was removed, scleral applicator with radioactive ${ }^{125}$ I was placed in and seamed on the sclera surface, then the conjunctiva was sutured. The applicator was removed under anesthesia after achieving certain radiation dose.

\section{Observational indexes}

Routine indirect fundus examination and ophthalmic A/B ultrasonic examination were conducted before every admission for new chemotherapeutical course. The decision on whether the therapeutic regimen should be continued or not was made according to the progression of the disease. Ophthalmic A/B ultrasonic examination was conducted after treatment, tumor control condition was observed, maximum basal tumor diameter, tumor thickness and other cancer-related data were recorded. The previous programs were reexamined once a month after treatment, every three months after six months, every six months after one year, once a year after two years, till puberty. The occurrence of adverse reactions was observed during and after the treatment.

\section{Evaluation criteria}

Degeneration of tumor refers to Tumor volume was reduced or significantly calcified compared with the situation before treatment. Invalidity, progress, recurrence and metastasis refer to new focus, continuous growth of tumor, invasion of vitreum and retina, dissemination and metastasis to other parts.

\section{Statistical analysis}

The data was analyzed by SAS 9.3 statistical package. Maximum tumor basal diameter and tumor thickness before and after treatment were conducted self control t-test. Univariate analysis was conducted to analyze factors affecting the prognosis, and rates in each group were compared by $\chi^{2}$ test. Variates with the result of $P<0.2$ in univariate analysis were analyzed by multi-factor logistic stepwise regression. The difference was statistically significant when $P<0.05$.

\section{Results}

\section{Clinical efficacy of chemoreduction combined with topical} treatment

All children received 2 6 courses of chemoreduction, (4.5 \pm 0.8$)$ courses on average; 17 eyes from 13 children were treated by chemoreduction combined with cryotherapy or TTT while 5 eyes from 4 children by chemoreduction combined with ${ }^{125}$ I episcleral plaque brachytherapy. The tumor size significantly decreased and partially calcified after 2 courses of chemotherapy, and tumor depauperated significantly and was stably controlled after 6 courses of chemotherapy combined with topical treatment (Figure 1 3). Among the 22 affected eyes, 4 eyes developed secondary glaucoma due to tumor progression or tumor necrosis and were finally removed, while 18 eyes were retained, with the eye retention rate being $81.82 \%$ (18/22). Among the 18 retained eyes, 7 were 


\section{$\gamma$}
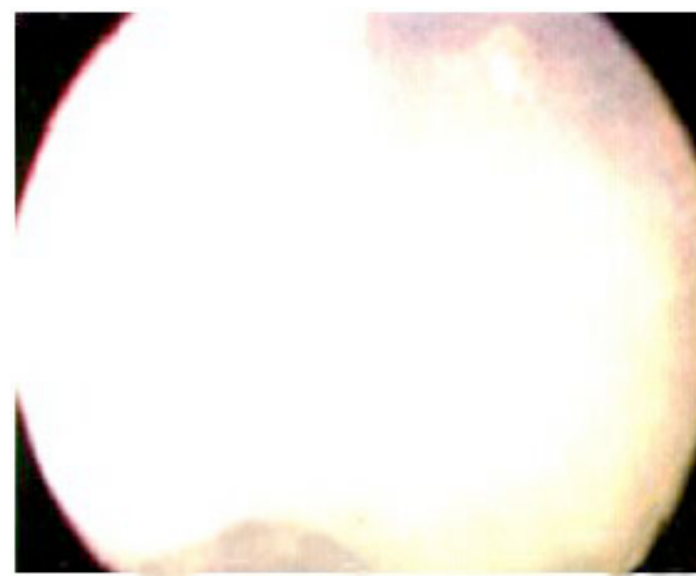

Figure 1. Color Image of Ocular Fundus before Treatment

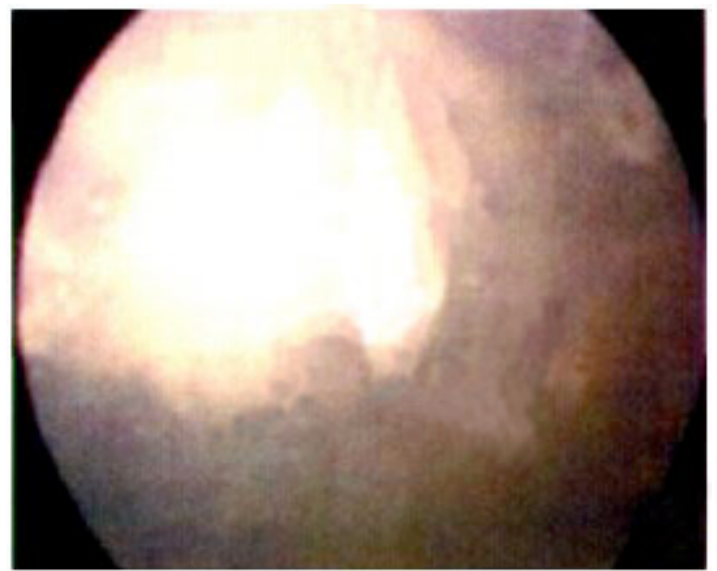

Figure 2. Color Image of Ocular Fundus after 2 Courses of Chemotherapy

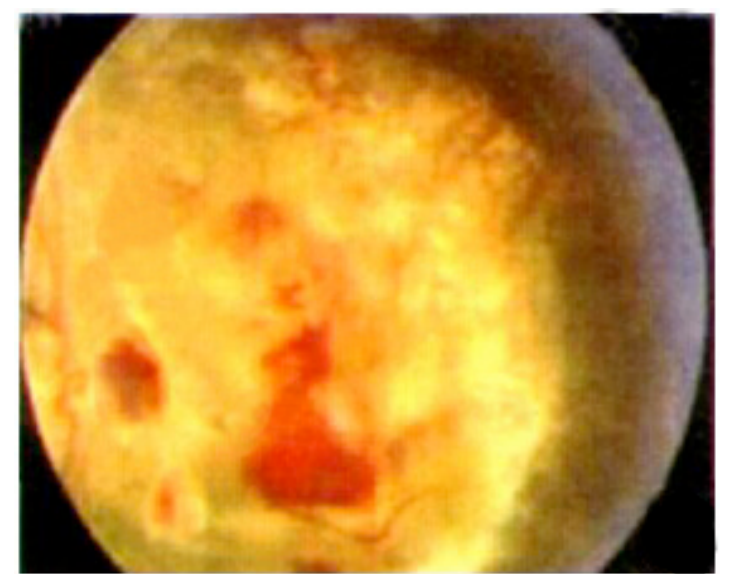

Figure 3. Color Image of Ocular Fundus after 6 Courses of Chemotherapy Combined with Topical Treatment

restored or with good vision, 3 with poor vision unable to be improved since the original tumors located in macula and failed to absorb subretinal fluid for a long time, and the rest 8 didn't gain good vision due to other reasons. Postoperative follow-up time was 7 34 months, (18.6 \pm 5.2) months on average. During the follow-up period, all children had tumor degeneration, while 7 recurred, with recurrent rate being $41.18 \%$ (7/17), among which 4 were controlled after supplementing or appending cryotherapy or TTT treatment, and the eyeballs of another 3 children
Table 1. Changes of Tumor Basal Diameter and Thickness Before and After Treatment

\begin{tabular}{|c|c|c|c|c|}
\hline & \multicolumn{2}{|c|}{$\mathrm{X} \pm \mathrm{s}$} & $\mathrm{t}$ & $P$ \\
\hline \multicolumn{5}{|c|}{ Tumor basal diameter $/ \mathrm{mm}$} \\
\hline Before treatment & \multirow{2}{*}{\multicolumn{2}{|c|}{$\begin{array}{r}12.86 \pm 4.21 \\
7.14 \pm 3.98\end{array}$}} & \multirow[t]{2}{*}{4.63} & \multirow[t]{2}{*}{$<0.0001$} \\
\hline After treatment & & & & \\
\hline \multicolumn{5}{|l|}{ Tumor thickness/mm } \\
\hline Before treatment & \multicolumn{2}{|c|}{$7.85 \pm 3.47$} & \multirow[t]{2}{*}{3.36} & \multirow[t]{2}{*}{0.0017} \\
\hline After treatment & \multicolumn{2}{|c|}{$4.54 \pm 3.06$} & & \\
\hline \multicolumn{5}{|c|}{ Table 2. Prognostic Factors - Univariate Analysis } \\
\hline & $\begin{array}{c}\mathrm{VA} \geq 6 / 12 \\
{[\mathrm{n}(\%)]}\end{array}$ & $\begin{array}{r}\mathrm{VA}<6 / 12 \\
{[\mathrm{n}(\%)]}\end{array}$ & $\chi^{2}$ value & $P$-value \\
\hline \multicolumn{5}{|l|}{ Number of tumor } \\
\hline Single & $2(9.09)$ & $7(31.82)$ & 3.3157 & 0.0686 \\
\hline Mutiple & $5(22.73)$ & $8(36.36)$ & & \\
\hline \multicolumn{5}{|c|}{ Location of tumor } \\
\hline Outside the macula & $3(13.64)$ & $2(9.09)$ & 2.3689 & 0.1238 \\
\hline Macula & $4(18.18)$ & $13(59.09)$ & & \\
\hline \multicolumn{5}{|l|}{$\%$ disc overhang } \\
\hline $0 \%$ & $6(47.06)$ & $2(9.09)$ & 10.8308 & 0.0044 \\
\hline $30 \%$ & $0(0)$ & $1(4.55)$ & & \\
\hline $60 \%$ & $0(0)$ & $0(0)$ & & \\
\hline $100 \%$ & $1(4.55)$ & $12(54.55)$ & & \\
\hline \multicolumn{5}{|c|}{ Tumor basal diameter before treatment } \\
\hline$<10 \mathrm{~mm}$ & $6(27.27)$ & $2(9.09)$ & 10.8054 & 0.0010 \\
\hline$\geq 10 \mathrm{~mm}$ & $1(4.55)$ & $13(59.09)$ & & \\
\hline \multicolumn{5}{|c|}{ Tumor thickness before treatment } \\
\hline$<6 \mathrm{~mm}$ & $7(31.82)$ & $7(31.82)$ & 5.8667 & 0.0154 \\
\hline$\geq 6 \mathrm{~mm}$ & 0 & $8(36.36)$ & & \\
\hline
\end{tabular}

were finally removed.

Changes of tumor basal diameter and tumor thickness before and after treatment

Tumor basal diameter decreased by $43.86 \%$ on average and tumor thickness decreased by $42.54 \%$ on average after treatment, and the differences were statistically significant when compared with treatment before, as shown in Table 1.

\section{Occurrence of adverse reactions}

During chemotherapeutic treatment, all children appeared different degrees of hair loss, in which $70.59 \%$ (12/17) had different degrees of anorexia, nausea, emesis and other gastrointestinal reactions, 5.88\% (1/17) appeared neutropenia, and $11.76 \%(2 / 17)$ were seriously infected. No leukemia, hepatic and renal functional lesion, hearing impairment and other serious chemotherapeutic toxicity reactions were observed.

\section{Analysis of prognostic factors}

The prognosis was better to tumors locating outside the macula, with less number, were farther from optic disc, and with smaller tumor basal diameter and thickness before treatment. Univariate analysis showed that whether the visual acuity (VA) after treatment was greater than 6/12 was only associated with the tumor basal diameter, tumor thickness and the distance to optic disc, as shown in Table 2. Further logistic regression analysis indicated that only tumor basal diameter before treatment had significant effect on the prognosis $\left(\chi^{2}=17.1183, P<0.0001\right)$. 


\section{Discussion}

$\mathrm{RB}$ is a common intraocular malignant tumor in infants (Khaenam et al., 2012; Khan et al., 2013; Li et al., 2013; Maiti et al., 2013; Srivastava et al., 2013; Romus et al., 2013). Some children have been sick at birth and appear vision loss and even blindness in the early stage. Since the vast majority of infantile patients can't express for themselves, it's difficult to early detect RB. When leukocoria or strabismus, proptosis and other obvious symptoms appear, the disease often has developed into the advanced stage (Atchaneeyasakul et al., 2009). Traditional therapeutic regimens include ophthalmectomy, evisceration, external beam radiotherapy and systemic chemotherapy. In the past, chemotherapy was seldom applied to intraocular RB due to the low efficacy, adverse reactions and systemic complications. The international clinical treatment of RB has been more conservative in recent years, and some scholars use chemotherapy regimen combined with topical cryotherapy, laser and other topical therapies to treat intraocular RB, which greatly improved the eye retention rate and QOL of children (Lambert et al., 2008).

Chemoreduction reduces the tumor volume through chemotherapeutic treatment to continue the topical treatment within more limited location and cause minor lesions. Chemoreduction can effectively improve the survival rate under the premise of retaining the eyes (Aerts et al., 2013). It was reported that the cure rate reached up to $75 \%$ after 6 courses of chemotherapy (carboplatin, etoposide, vincristine and cyclophosphamide as )combined with laser, thermotherapy and other topical therapies (Künkele et al., 2013). The research of Berry et al showed that the cure rate of binoculus RB treated by chemoreduction was $47 \%$, with the survival time of $82 \%$ of the patients was more than 12 months and $68 \%$ more than 60 months (Berry et al., 2013). The application of chemoreduction significantly increase the retention rate of RB patients, but new RB still occur in $24 \%$ of patients during or after the treatment, most of which are in infancy or have a family history of the disease (Shields et al., 2003). Turaka et al pointed out that chemotherapies consisting of carboplatin, etoposide and vincristine could induce second primary cancer in $4 \%$ of children (Turaka et al., 2012). And another study has shown that $47 \%$ of patients need external beam radiotherapy and $53 \%$ require ophthalmectomy after 5 years of pure chemotherapy (Shields et al., 2002).

Topical treatments of RB consist of laser photocoagulation, TTT, cryotherapy and episcleral plaque brachytherapy, most of which are suitable for the treatment of small tumors, especially tumors with reduced volume after chemoreduction treatment. Topical treatments for each tumor should be carried out repeatedly and simultaneously in every stage during the chemoreduction treatment. Reasonable combined therapy can effectively inhibit tumor, retain the eyeball and save the vision (Kim et al., 2010; Shin et al., 2010). it was reported that the eye retention rate was $67 \%$ after treatment of chemoreduction combined with topical therapies such as cryotherapy, brachytherapy or laser photocoagulation, significantly higher than single chemotherapy (Balwierz et al., 2010).

The results of this study indicated that chemoreduction combined with TTT, cryotherapy or ${ }^{125} \mathrm{I}$ episcleral plaque brachytherapy could effectively control RB in a short term with the eye retention rate being $81.82 \%(18 / 22)$ and 7 affected eyes restoring or maintaining good vision, which could significantly improve the QOL of children. However, the recurrence rate was $41.18 \%$ (7/17) after treatment, of which $57.14 \%$ (4/7) were controlled after receiving supplementary or additional cryotherapy or TTT treatment, suggesting that the close follow-up, in time detection and topical supplementary therapy were a important methods to control the disease and improve eye retention rate.

To sum up, this study indicates that chemoreduction combined with topical therapy can effectively control the disease and improve the QOL of children. Logistic regression analysis shows that tumor basal diameter before treatment is an independent risk factor for prognosis. However, the effective sample size of this study is limited, and the curative effect, adverse reactions and prognostic factors of chemoreduction combined with topical treatment need to be confirmed through more large-scale studies.

\section{References}

Atchaneeyasakul L, Wongsiwaroj C, Uiprasertkul M, et al (2009). Prognostic factors and treatment outcomes of retinoblastoma in pediatric patients: a single-institution study. Jap J Ophthalmol, 53, 35-9.

Aerts I, Sastre-Garau X, Savignoni A, et al (2013). Results of a multicenter prospective study on the postoperative treatment of unilateral retinoblastoma after primary enucleation. J Clin Oncol, 31, 1458-63.

Balwierz W, Pietrys D, Romanowska-Dixon B, et al (2010). Results of combined chemotherapy and local ophtalmic therapy for intraocular retinoblastoma. Przegl Lek, 67, 404-8.

Berry JL, Jubran R, Kim JW, et al (2013). Long-term outcomes of Group D eyes in bilateral retinoblastoma patients treated with chemoreduction and low-dose IMRT salvage. Pediatr Blood Cancer, 60, 688-93.

Huang D, Zhang Y, Zhang W, et al (2013). Study on clinical therapeutic effect including symptoms, eye preservation rate, and follow-up of 684 children with retinoblastoma. Eur J Ophthalmol, 23, 532-8.

Khan ZN, Sabir M, Kayani MA, et al (2013). Acetylation of retinoblastoma like protein2 $(\mathrm{Rb} 2 / \mathrm{p} 130)$ in tumor tissues. Asian Pac J Cancer Prev, 14, 2255-8.

Kim JM, Kim JH, Kim SJ, et al (2010). Visual prognosis of retinoblastoma in the posterior pole treated with primary chemotherapy plus local treatments. Korean J Ophthalmol, 24, 347-52.

Künkele A, Jurklies C, Wieland R, et al (2013). Chemoreduction improves eye retention in patients with retinoblastoma: a report from the German Retinoblastoma Reference Centre. Br J Ophthalmol, 97, 1277-83.

Lambert MP, Shields C, Meadows AT (2008). A retrospective review of hearing in children with retinoblastoma treated with carboplatin-based chemotherapy. Pediatr Blood Cancer, 50, 223-6.

Li J, Xu ZW, Wang KH, et al (2013). Networks of MicroRNAs and genes in retinoblastomas. Asian Pac J Cancer Prev, 14, 6631-6.

Peterson EC, Elhammady MS, Quintero-Wolfe S, et al (2011). 
Selective ophthalmic artery infusion of chemotherapy for advanced intraocular retinoblastoma: initial experience with 17 tumors: Clinical article. J Neurosurg, 114, 1603-8.

Shields CL, Honavar SG, Meadows AT, et al (2002). Chemoreduction plus focal therapy for retinoblastoma: factors predictive of need for treatment with external beam radiotherapy or enucleation. Am J Ophthalmol, 133, 657-64.

Shields CL, Shelil A, Cater J, et al (2003). Development of new retinoblastomas after 6 cycles of chemoreduction for retinoblastoma in 162 eyes of 106 consecutive patients. Arch Ophthalmol, 121, 1571-6.

Shields CL, Shields JA (2010). Retinoblastoma management: advances in enucleation, intravenous chemoreduction, and intra-arterial chemotherapy. Curr Opin Ophthalmol, 21, 203-12.

Shin JY, Kim JH, Yu YS, et al (2010). Eye-preserving therapy in retinoblastoma: prolonged primary chemotherapy alone or combined with local therapy. Korean J Ophthalmol, 24, 219-24.

Srivastava V, Patel B, Kumar M, et al (2013). Cyclin D1, retinoblastoma and $\mathrm{p} 16$ protein expression in carcinoma of the gallbladder. Asian Pac J Cancer Prev, 14, 2711-5.

Turaka K, Shields CL, Meadows AT, et al (2012). Second malignant neoplasms following chemoreduction with carboplatin, etoposide, and vincristine in 245 patients with intraocular retinoblastoma. Pediatr Blood Cancer, 59, 121-5. 